\title{
Medizinethik in Zeiten des Moralismus
}

\author{
Ralf J. Jox
}

Angenommen: 3. August 2021

(C) Springer-Verlag GmbH Deutschland, ein Teil von Springer Nature 2021

Die gegenwärtige Coronapandemie kann als Brennglas begriffen werden, das gesellschaftliche Trends bündelt und uns auf engem Raum vor Augen führt, welche Merkmale und Herausforderungen unsere Zeit kennzeichnen. Und so weist uns die moralisch aufgeheizte, aufgeregte Stimmung rund um Lockdown, Triage, Impfpflicht und Covid-Zertifikate auf eine Entwicklung hin, die bereits in den Jahren vor Corona immer häufiger beschrieben wurde: einen grassierenden Moralismus (Grau 2017; Hübl 2019; Kissler 2020; Lübbe 2019; Neuhäuser und Seidel 2020).

So sehr die moralischen Urteile und Inhalte selbst oft legitim und hoch relevant sind, so sehr irritiert doch mehr und mehr die Vehemenz moralischer Verurteilungen, der verletzende Ton im Umgang miteinander und die handgreiflichen, ja existenziellen Konsequenzen, welche all das mitunter hat. Eine Politikerin drückt sich linkisch aus und muss öffentlich zu Kreuze kriechen, ein Sportler verletzt die Sprachetikette und verliert seine Sponsorenverträge, eine Künstlerin wird wegen missverstandener satirischer Aussagen von Veranstaltungen ausgeladen. Wer hat sich nicht schon dabei ertappt, dass er oder sie aus Angst vor einem Shitstorm manch eine öffentliche Meinungsäußerung unterlassen hat. Moralisch inkriminierte Wörter werden aus literarischen Klassikern getilgt, Denkmäler posthum verfemter Persönlichkeiten werden von Bürgern eigenhändig zerstört. Intellektuelle mit missliebigen Meinungen jeder politischen Couleur, weit innerhalb des verfassungsgemäßen Spektrums, werden von Tagungen ausgeschlossen. Podcasts, Blogs und Posts im Internet werden gelöscht und die Urheber gesperrt, sobald sie Meinungen vertreten, die vom Mainstream abweichen, egal in welche Richtung.

Prof. Dr. med. Dr. phil. Ralf J. Jox $(\bowtie)$

Institut des humanités en médecine, Centre Hospitalier Universitaire Vaudois (CHUV) und Universität Lausanne, Avenue de Provence 82, 1007 Lausanne, Schweiz

E-Mail: ralf.jox@chuv.ch 
Schon die Tatsache, dass wir für diese Prozesse eigene Schlagworte erfunden haben, unterstreicht ihre Relevanz in der heutigen Gesellschaft: political correctness, cancel culture, wokeness oder Moralismusfalle, um nur einige $\mathrm{zu}$ nennen. Über die Ursachen dieses Moralismus werden erste Mutmaßungen diskutiert: Er scheint offenbar eng verbunden mit der Identitätspolitik, dem Streben nach kollektiver Zugehörigkeit in einer Welt der Unübersichtlichkeit und Heimatlosigkeit, in der traditionelle Ordnungs- und Orientierungskategorien wie Religion, Partei, Beruf und Familie an Bedeutung verloren haben. Die internetbasierten Kommunikationsformen scheinen den Trend zum Moralismus noch zu fördern, indem sie das intuitive, binäre Urteilen über andere durch ihre fest eingebauten Bedienungselemente (Likes, Retweets, Kommentare) bahnen.

Aber was genau ist unter Moralismus zu verstehen? Ich möchte ihn definieren als die Inflation und infantil-impulsive Praxis moralischen Urteilens über Andere. Damit sind drei wesentliche Elemente zu differenzieren: Erstens: Das inflationäre Element des Moralismus besteht darin, dass moralische Kategorien auf bislang außermoralische Gegebenheiten angewandt werden. Es gibt keinen moralfreien Raum mehr, jedes Spiel, jede sportliche Tätigkeit, jede private Aktivität bis hin zum Speiseplan und zur Sprachform wird dramatisierend mit Moral aufgeladen. So ging es etwa bei der Übersetzung des Gedichts der US-amerikanischen Poetin dunkler Hautfarbe Amanda Gorman nicht mehr um die Qualität der Übersetzung, sondern auf einmal wurde skurrilerweise die Hautfarbe der Übersetzerin zum moralischen Bewertungskriterium. Auch die Differenz zwischen Moral und Recht löst sich auf: Wenn etwa ein Krankenpfleger in seiner Freizeit mit dem Auto zu schnell über die Landstraße fährt und eine Ordnungswidrigkeit begeht, sieht er sich in manchen Gegenden bereits einem berufsethischen Verfahren gegenüber.

Zweitens: Das infantile Element des Moralismus bezieht sich darauf, dass die Weise moralischen Urteils über andere nach einem simplen Schwarz-Weiß-Schema funktioniert und damit auf das moralpsychologische Entwicklungsniveau von Kleinkindern regrediert. Der Dualismus zwischen Gut und Böse, Freund und Feind, genährt durch zahlreiche Hollywood-Märchen, findet sich leider auch allzu oft in moralischen Alltagsurteilen. Nicht nur die Medien erweisen sich als terribles simplificateurs und fragen auf platte Weise, ob man für oder gegen Sterbehilfe, für oder gegen die Gentechnik, für oder gegen die Impfpflicht sei. In dieser schwarzweißen Moralwelt gibt es keine Grauschattierungen mehr, geschweige denn Farben. Diese Art der Komplexitätsreduktion ist verführerisch, aber sie geht an der Wirklichkeit vorbei.

Drittens: Das impulsive Element des Moralismus schließlich äußert sich darin, dass der moralische Diskurs nicht argumentativ-rational, sondern impulsiv-emotional und ad personam geführt wird. Es werden nicht Behauptungen und Begründungen ausgetauscht, geprüft und abgewogen, sondern Vorwürfe, Beschuldigungen und Symbolhandlungen genutzt. Der Moralist operiert mit moralischen Gefühlen, stiftet Schuld und Scham. Ein Beispiel dafür ist, sämtliche ,alten, weißen Männer" in die Schamecke zu stellen. Auch in der Medizin mache ich immer öfter die bedenkliche Erfahrung, dass Patienten kulpabilisiert werden und Fachkräfte sich empört weigern, Patienten erneut zu behandeln, wenn diese zum wiederholten Mal wegen Alkoholkonsums eine Leberkrise erleiden oder mangels Medikamentenad- 
härenz wieder stationär behandelt werden müssen. Die impulsive Abwehr und der moralische Abscheu helfen aber in der Regel nicht dabei, ein moralisches Problem zu lösen, sondern rufen lediglich bei der Gegenseite ähnlich starke Gefühle von Wut, Trotz, Beleidigung und Hass hervor, so dass sich eine emotionale Spirale aufschaukeln kann, was etwa in Shitstorms in den social media eindrücklich beobachtet werden kann. Das Unmenschliche am Moralismus liegt darin begründet, dass im Kern nicht mehr gegen Meinungen, sondern gegen Personen vorgegangen wird. Wer vorgibt, das moralisch Gute zu betreiben, in seinem eigenen kommunikativen Vollzug aber das moralisch Gute ignoriert und diskursethische Prinzipien verletzt, der begeht einen performativen Selbstwiderspruch.

Der Moralismus zieht seine verführerische Kraft aus einer dreifachen Befriedigung des postmodernen Menschen: Er befriedigt dessen Suche nach eindeutiger Ordnung in einer unübersichtlichen Welt (kognitive Befriedigung); er gibt ihm das Gefühl, inmitten einer Welt insulärer Individuen in der wohligen Gemeinschaft Gleichgesinnter geborgen zu sein (affektive Befriedigung); und er befreit ihn von der Wahl zwischen tausenderlei Lebensmöglichkeiten und Sinnangeboten in unserer pluralen Gesellschaft, indem er ihm ein vermeintlich absolutes, unangreifbares moralisches Gut als Sinn vorspiegelt (volitionale Befriedigung).

Nachdem ich nun umrissen habe, worin der Moralismus besteht und worin seine Attraktivität wurzelt, drängt sich die Frage auf, weshalb der Moralismus überhaupt ein Problem darstellt und wieso er zu vermeiden ist. Da der Moralismus eine übertriebene und fehlgeleitete Form moralischer Tätigkeit darstellt, erweist er letztlich der eigenen Sache einen Bärendienst und erreicht in der Regel das Gegenteil dessen, was er beabsichtigt. Anstatt die legitimen moralischen Inhalte zu verwirklichen, provoziert er Reaktanz, Spaltung und nicht selten sogar Gewalt. Sein Ziel erreicht er auch deshalb nicht, weil er schlicht die gesellschaftliche und anthropologische Realität in ihrer Komplexität und Differenziertheit nicht in den Blick nimmt und damit aus pragmatischer Sicht auf Dauer scheitern muss. Weiterhin schränkt der Moralismus die individuelle und kollektive Freiheit ein, da er den Menschen vermeintliche Entweder-Oder-Alternativen aufdrängt, einen inneren Zensor installiert und sogar die Ausübung von Menschenrechten wie dem Recht auf freie Meinungsäußerung gefährdet. Dem Moralismus wohnt eine fatale Tendenz zu Intoleranz und Totalitarismus inne, welche häufig dadurch verstärkt wird, dass ein bestimmter moralischer Wert absolut gesetzt und als Heilslehre verfolgt wird. Das langfristig zu befürchtende Ergebnis ist die Abschaffung der offenen Gesellschaft und Demokratie, wie es Karl Raimund Popper 1945 luzide analysiert hat (Popper 2003).

Nun liegt für den Leser dieser Zeitschrift gewiss die Frage auf der Zunge, ob wir mit dem Bad des Moralismus nicht auch das Kind der Ethik ausschütten. Nichts könnte falscher sein als diese Vorstellung, denn die Ethik ist das genaue Gegenteil des Moralismus, gleichsam das Kontrastprogramm im Umgang mit moralischen Fragen. Ethik ist die rationale Reflexion von Moral, die nach Gründen sucht, um moralische Urteile, Entscheidungen und Handlungen im intersubjektiven Diskurs zu verantworten. Sie verhält sich in allen drei oben genannten Punkten antipodisch zum Moralismus: 1) Ethik differenziert sorgfältig moralische und außermoralische Kategorien und bewahrt die Moral damit vor einer inflationären Entwertung. Schon die stoische Ethik der Antike hatte erkannt, dass es neben Gut und Böse auch die 
adiaphora gibt, die sittlich neutralen Phänomene. 2) Ethik nutzt, im sokratisch-philosophischen Sinne, den radikalen Zweifel und das stete Hinterfragen vermeintlicher Gewissheiten, um die tückische Selbstgerechtigkeit zu entlarven, die trügerische Scheinerkenntnis zu vermeiden und der facettenreichen Wirklichkeit gerechter zu werden. Dabei eröffnet Ethik oft die Erkenntnis, dass es einen Korridor plausibler, legitimer und gerechtfertigter Verhaltensweisen gibt, nicht nur den einen, einzig seligmachenden Weg. 3) Ethik intendiert, in der Tradition der Aufklärung, die Mündigkeit des Individuums, damit es auf der Basis praktischer Rationalität autonom und verantwortungsvoll handeln kann. Zusammengefasst lässt sich sogar behaupten, dass Moralismus und Ethik einander wechselseitig ausschließen: Wer moralistisch agiert, macht Ethik obsolet, und wer ethisch argumentiert, überwindet eo ipso den Moralismus.

Das bedeutet aber mitnichten, dass Ethiker im Allgemeinen und Medizinethiker im Besonderen vor der Versuchung des Moralismus gefeit wären. So sollte Medizinethik sich zum Beispiel nicht darin erschöpfen, moralpragmatisch das Gute in der Medizin durchzusetzen, sondern muss dies immer auch argumentativ begründen, damit es anderen einsichtig werden kann. Appelle und Memoranden mögen zuweilen hilfreich und nötig sein, aber sie dürfen nicht zum Gutmenschen-Aktivismus, zum fanatischen Morallobbyismus oder zum larmoyanten Lamento verkommen. Das gesteigerte mediale Interesse an Medizinethikern ist eine große Chance für unser Fach, aber es sollte uns nicht dazu verleiten, uns als autoritative Moralexperten, mit erhobenem Zeigefinger auf der Talkshow-Couch, vor den Karren des Moralismus spannen zu lassen. Auch die von Politikern emsig eingesetzten Ethikkommissionen müssen aufpassen, dass sie ihre Stellungnahmen nicht als dezidierte Dekrete abfassen, die expertokratisch das Gute und Richtige verkünden, sondern als Tableau von Argumentationen, welche zum kritischen Weiterdenken anregen. Sache der Ethiker ist es nicht, den Daumen zu heben oder zu senken, sondern dem ubiquitären Abstimmungsdiskurs einen Argumentationsdiskurs entgegenzusetzen.

In diesem Sinne möchte auch die Zeitschrift „Ethik in der Medizin“ in ihrem 33. Jahrgang weiterhin eine Plattform für einen solchen Argumentationsdiskurs sein. Die vergangenen fünf Jahre über hat Thomas Schramme die Zeitschrift als federführender Schriftleiter genau auf diesem Weg vorangebracht, sie zur Philosophie hin geöffnet und durch die Ermöglichung englischsprachiger Beiträge internationalisiert (Schramme 2016). Als sein Nachfolger danke ich ihm ganz herzlich und möchte mich dafür engagieren, dass die Zeitschrift ein Forum kontroverser, konstruktiver Debatten bleibt, das in Zukunft hoffentlich noch stärker als bisher in die Medizin, in die Wissenschaft und in die Gesellschaft hineinwirkt. Dafür danke ich schon jetzt der Redaktion, der gesamten Schriftleitung, den Beiratsmitgliedern, dem Verlag und der Akademie für Ethik in der Medizin, allen künftigen Autorinnen und Autoren und ganz besonders Ihnen, den treuen Leserinnen und Lesern von „Ethik in der Medizin“.

Interessenkonflikt R.J. Jox ist federführender Schriftleiter der Zeitschrift „Ethik in der Medizin“.

\section{Literatur}

Grau A (2017) Hypermoral. Die neue Lust an der Empörung. Claudius, München 
Hübl P (2019) Die aufgeregte Gesellschaft: Wie Emotionen unsere Moral prägen und die Polarisierung verstärken. Bertelsmann, München

Kissler A (2020) Die infantile Gesellschaft. Wege aus der selbstverschuldeten Unreife. HarperCollins, Hamburg

Lübbe H (2019) Politischer Moralismus. Der Triumph der Gesinnung über die Urteilskraft. LIT, Münster Neuhäuser C, Seidel C (Hrsg) (2020) Kritik des Moralismus. Suhrkamp, Berlin

Popper KR (2003) Die offene Gesellschaft und ihre Feinde. Band I und II. Mohr Siebeck, Tübingen

Schramme T (2016) Philosophie und Medizinethik. Ethik Med 28:263-266 